Research Article

\title{
Data Analysis for Predictive Maintenance of Servo Motors
}

\author{
Oguz Girit $\mathbb{D}^{1},{ }^{1}$ Gurcan Atakok $\mathbb{D}^{1},{ }^{1}$ and Sezgin Ersoy $\mathbb{D}^{2}$ \\ ${ }^{1}$ Marmara University, Faculty of Technology, Department of Mechanical Engineering, Istanbul 34722, Turkey \\ ${ }^{2}$ Marmara University, Faculty of Technology, Department of Mechatronics Engineering, Istanbul 34722, Turkey \\ Correspondence should be addressed to Gurcan Atakok; gatakok@marmara.edu.tr
}

Received 12 June 2020; Revised 20 July 2020; Accepted 20 August 2020; Published 11 September 2020

Academic Editor: Chaoqun Duan

Copyright $\odot 2020$ Oguz Girit et al. This is an open access article distributed under the Creative Commons Attribution License, which permits unrestricted use, distribution, and reproduction in any medium, provided the original work is properly cited.

\begin{abstract}
Vibration and temperature data of a servo motor are analyzed with PLC which is widely used in the industry. With this system, power supply can be detected on the servo motors. In this way, undesirable situations such as disruptions in production and productivity loss can be prevented from occurring. It is an important problem for businesses to detect malfunctions that may occur in servo motor dysfunction. Previously, methods such as ultrasonic sound measurements, thermal cameras, endoscopy equipment, and energy analysis have been used and discussed in the literature. Our study offers a PLC-based vibration and temperature measurement system designed as a solution of this problem. In this system, vibration and temperature measurements were made while the servo motor was kept running. These measurements were measured with or without load, considering the operating ranges of the servo motor, and the compatibility of the data was evaluated.
\end{abstract}

\section{Introduction}

One of the biggest problems encountered in the automation sector is the loss suffered in production when the product dysfunctions. In order to prevent the unexpected malfunctions from occurring during the intensive production periods, the maintenance system must be well managed and organized. The implementation of maintenance activities is very important for the smooth operation of machines and work processes that operate continuously $[1,2]$. In such cases, it is possible to work with an external company to carry out the relevant maintenance work [3]. Briefly, maintenance activity is a planned and programmed movement that all the partitions in the firm create in an organized manner to maintain the functions of the systems in the most efficient way [4]. Research carried out on the efficiency of maintenance work shows that $33 \%$ of the maintenance expenses are unnecessary or wasted due to disruption of their periodic maintenance $[5,6]$. While maintenance strategy is created, for this reason, the selection of a business method that suits the maintenance requirements constitutes great importance [7]. Failure can occur while production is intensive. Maintenance and repair to be made during this process can cause high costs. Since the application of this method determines the malfunctions in advance, costs are minimized [6].
Even if the maintenance work is carried out in a comprehensive manner, it may become stereotyped over a long period, and it may not be possible to achieve the desired benefits proportional to the experience of the maintenance technician. Among the types of maintenance, predictive care occupies the last position, with an application rate of $2 \%$. This shows that the predictive care type has been ignored although it provides many benefits for companies because it can foresee and prevent failure before the equipment malfunction occurs [8]. The origin and the development of malfunctions learned from the analyses made with the data obtained can be used in high capacity use of engines and in avoiding shutdowns caused by timeless failures. The predictive care applications are measurement, analysis, and, respectively, repair [9]. The predictive care uses the vibration measurement tool, ultrasonic sound measurement tool, thermal cameras, endoscopy equipment, and energy analyser tools. Benefits of predictive care are the increased life cycle of equipment, estimation of maintenance time, prevention of labour loss, quality, and more efficient use [10-12]. Although the benefits of predictive care are known, it has been determined that the proportion of firms that determine their malfunctions by applying fractional care in the world is $0.04 \%$ [13].

The systems used to determine the malfunction of products such as electric motors, generators, and transformers commonly 
used in the industry measure voltage and current signals to determine the malfunction status of related products [14, 15]. Depending on the operating conditions and the characteristics of the points to be measured using various parameters that characterize the behaviour of systems at runtime to monitor the operating conditions and performances of the machine, the working performance of the machine under various physical quantities can be observed at certain intervals [16]. These measurements are carried out by employing methods. These methods include monitoring actual data, the load set and frequencies, performance curves during offline use, and use of the existing net worth $[17,18]$. However, the acquisition and processing of these data are disadvantageous due to the lack of an information boundary and the lack of continuity. System design and management of data traffic are important in the prevention and diagnosis of malfunctions. Writing-based system developments are among the attempted methods [19]. However, these system approaches are generally concentrated on vibration.

For predictive maintenance purposes, thermodynamic and dynamic analysis of a motor that operates between the attachment of the sharp ends of the cylinder, gamma type, free piston, and hot and cold welding temperature can be carried out by detecting the development of a running machine's failure [1] on asynchronous motors [2]. Also, real-time monitoring and evaluation of rotating machines can be done [20]. Today, some methods have been used to determine the friction problems of fixed and rotating parts. One of these methods is friction spectral analysis which is carried out by the measurement of the distribution of the characteristics of reaction. However, the disadvantage of this method is that the friction generating equipment produces noise in the current frequency band. A complete analysis of the temporary response of the rotor-stator interaction in which the friction process is represented by a linear product model (Coulomb friction), and the distribution of the cavity effects due to friction in the spiral vibrations which increases the stability of the system in the rubbing area, has been detected [21, 22].

Our study offers a PLC-based vibration and temperature measurement system designed for the solution of this problem. Vibration and temperature measurements were made while working on the servo motor made according to this system. These measurements were measured with and without load, while considering the operating ranges of the servo motor and while considering the compatibility of working with the graphical data analyses.

\section{Materials and Methods}

This system is designed to measure the vibration and temperature measurements in servo motors. As seen in Figure 1, the vibration values and temperature values of the surface area of the servo motor on the $X$ and $Y$ axes were controlled by PLC, which is widely used in the industry.

In this study, the automation systems (motor, moving elements, etc.) is used to measure the vibration and temperature values of the products to determine whether the products comply with the standards and to prevent malfunctions. The acceptance of vibration values obtained from

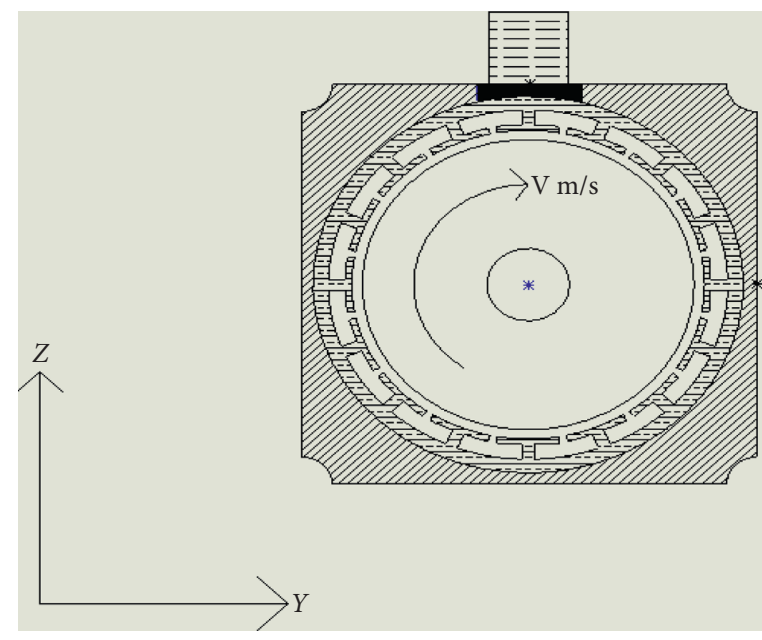

FIGURE 1: Servo motor working principle.

the measurements of the machines has been determined with the international ISO 2372 standard. This standard has been used to evaluate the vibration intensity of machines operating between $10-200 \mathrm{~Hz}$ [23]. In this study, measurements and evaluations were applied to servo motors between $10-50 \mathrm{~Hz}$ by connecting them to any machine. All the harmonic movements that occur in simple harmonic vibrations are repeated periodically. The magnitude of the forces required for the vibration to occur is proportional to the intensity of the vibration [24, 25]. Displacement, velocity, and acceleration are units of amplitude. The unit to be used during the measurement is decided upon the system's work value $\mathrm{Hz}$. The measurements done have been interpreted and evaluated according the ISO 2372 standards.

Temperature is a term connected to a system's molecule's average kinetic energy. It is a base magnitude and a scaler. As the temperature increases, the kinetic energy of the molecules also increases and they move faster; while temperature decreases, the molecules' kinetic energy decreases well and they move slower. If two or more objects are in contact, an energy transfer occurs from the hot objects to the cold objects until there is a thermal equilibrium [26]. Temperature measuring detectors, which are frequently used in industrial environments, are very important, because they determine the temperature range and process conditions made in industrial environments. These measuring systems are generally of low cost semiconductor (PTC-NTC and similar) materials. Today, analog temperature detectors such as NTC and PTC are also used alongside digital temperature detectors.

The operating algorithm of the system is shown in Figure 2. Press start to perform vibration and temperature analysis and select the servo motor model in the recipes. Measurement will start automatically after the recipe is selected. The measurement will be completed when the preset time has elapsed. If the servo motor is operating in accordance with normal operating conditions, the SCADA system will record the data and finish the operation. If the servo motor is not operating in accordance with normal operating conditions, the SCADA system will warn and the servo motor maintenance operation will be required. 


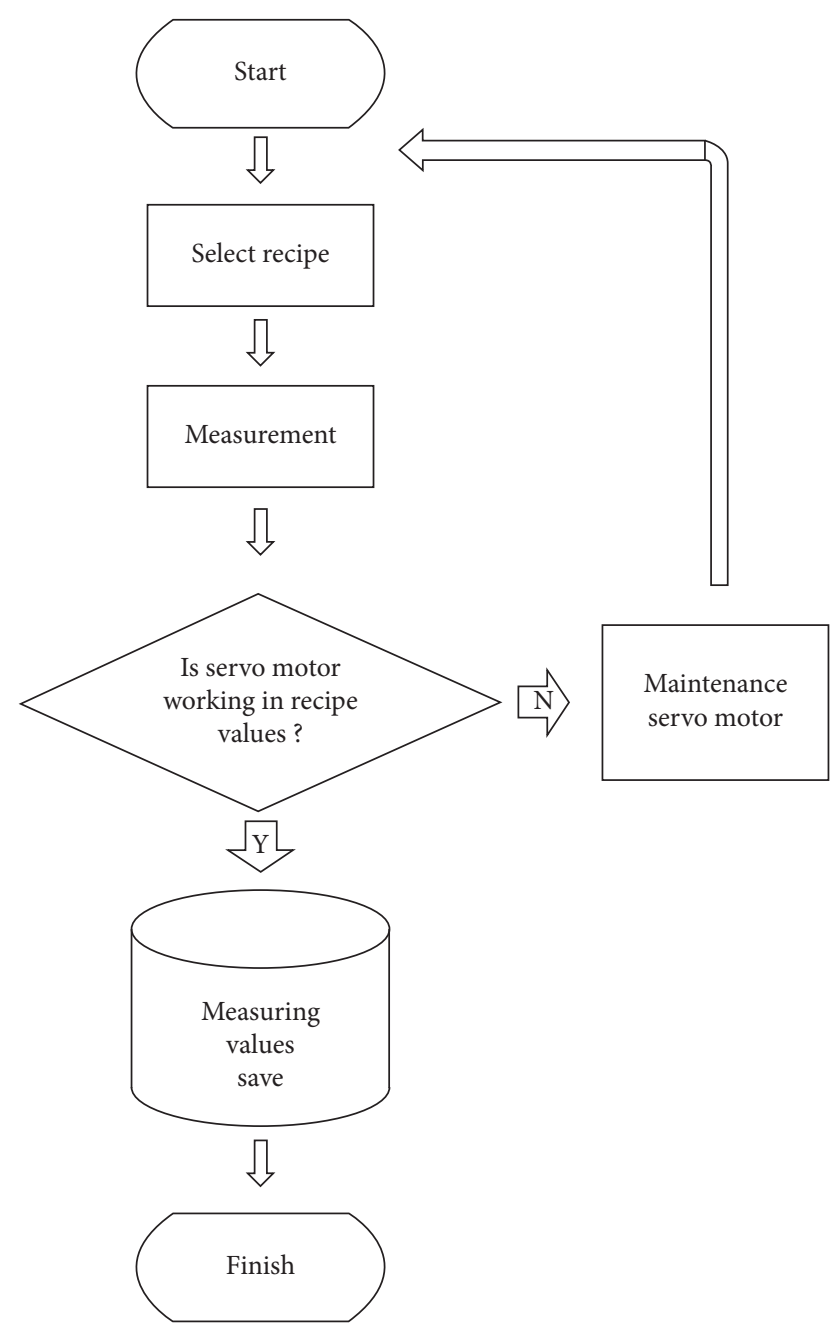

FIgURE 2: The operating algorithm of the system.

2.1. System Design. The system designed in this work is used to measure the vibration and temperature values of servo motors. When selecting a detector, the following aspects need be paid attention:

The reading sensitivity of the detector.

The minimum and maximum values to be measured.

The sensitivity limit against the highest temperature to be measured.

The reaction speed and the reading accuracy against the change of temperature in unit time.

The continuation time of determination and accuracy.

The restrictions of the environment.

The accuracy level of the application and the change in cost according to the way the detector has been mounted. As there are transducers that measure temperature without contact, temperature detectors usually work by being in contact with the surface that is going to be measured. The temperature detecting equipment consists of thermoelectric temperature elements and resistive temperature elements.
2.2. Vibration and Temperature Measurement. Vibration is expressed in two physical variables which are vibration frequency and intensity of vibration. The frequency of vibration is the number of vibration in a unit of time, and the unit is expressed in hertz $(\mathrm{Hz})$. The severity of vibration is the current strength that occurs in unit of time in the environment where the vibration occurs perpendicular to the energy which comes from the vibration, and the unit of the severity of vibration is $\left(\mathrm{W} / \mathrm{cm}^{2}\right)$ [27].

Analysis of the data obtained by measurement is important for maintenance and performance use. Accurate analysis of these data creates predictable data for machine failure. For example, the balancing of all forces on piston and rotating machines and the use of special montages decrease the stress. Likewise, the vibration characteristics of the system need to be understood, and the resonance condition analysis needs to be carried out to get an excellent working performance [28]. The goal here is to avoid resonance with the measurement of the vibrations created and the experiments. Moreover, thus decrease releases, many studies have been performed on this. As an example, the deduction below has been made about a ship's vibration in conclusion of the experiments made [29].

Alongside the measurements of vibration, the temperature measurements also carry importance to a large extent. Because it is a parameter that affects various properties and creates a deformation effect on materials, it is essential for the measurements to be done in specific periods to be controlled. Different temperature measurement devices can be designed by taking advantage of their various thermometric features in the measurement of materials' temperatures. Today, there are various temperature measurement devices that depend on the length, pressure, volume, electric resistance, electromotor force inside the electric circuit created by two different wires, and the changes of materials' external heat intensity. These along with devices usually measure by being in contact with the surface to be measured. Besides this method, there are devices used in measuring high temperatures that measure contact-free [16].

In an expertly designed algorithm, the number of transactions must be constant per data instance. Therefore, the total number of operations must be linear depending on $N$. In general, the processing time required for a collection is much shorter than the processing time required for a multiplication operation. Algorithms can be developed to make these complex operations quick and easy [30]. With this analysis, the signal can be seen in the frequency domain, and the frequency spectrum in the blocks can be calculated and displayed. Real-time data processing is used to calculate time, field signals, and the frequency domain signals obtained from these signals must also be higher than the data acquisition rate.

The control of vibration-temperature during operation and a suitable system for the servo motors to operate have been created. The system is run by the PLC. The sensors have been used to measure the temperature and the vibration values of the devices used in the systems are shown in Figure 3 . 


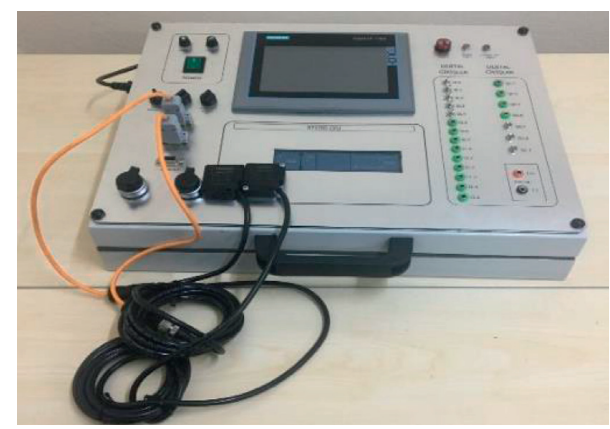

Figure 3: View of the designed system.

The vibration and temperature sensor code used in this process is QM42VT2. The vibration sensors have $X$ - and $Z$ axis indicators on the surface. When $X$ is parallel to the sensor, the $Z$ axis moves through the sensor on a plane. The $X$ axis is mounted on the same axis as the motor shaft or on it axially.

For best results, the sensor should be installed as close as possible to the motor mount. If this is not possible, the sensor must be mounted on a rigidly connected surface with the vibration characteristics of the motor. Using a surface with a cloth on or any another unstable mounting location to detect specific vibration characteristics can result in a reduced accuracy or capability.

2.3. Properties of the Designed Set. In order to evaluate the measured data in the designed measurement system, a Siemens S71200 PLC, a Siemens Comfort 9" operator panel with a SCADA system, two vibration and temperature monitors, and a Siemens Brand MODBUS card have been used to measure the vibration and temperature. The measured values have been transferred via the Modbus RTU protocol to the PLC. Five units have been used on the measurement system to control the on/off switch. Besides, a fuse has been added to protect the led system and the high supply voltage from alerting the alarm. The design of the system is shown in Figure 4.

The PLC program in the measuring system has been created by programming the Siemens Tia Portal Professional V14 SP1 software and the SCADA program installed in the operator panel with the Siemens Win CC Comfort V14 SP1 software. Modbus RTU and Ethernet protocol was created to ensure communication between the products used in the system.

\section{Results}

Through the system designed in this paper, a servo motor used in the industry has been implemented. The measurements have been made between 0-3000 and 3000-0 RPM. Besides, vibration and temperature analyses have been performed by running the motor with and without load.

3.1. 0 to 3000 RPM Unloaded. The servo motor, with the label information seen, has values increased from 0 RPM to 3000 RPM with no load, and the vibration and temperature values have been recorded for 60 seconds. The vibration data have been taken on the body both in the vertical and in the horizontal axes; the temperature data have also been taken from the body (see the graphs from Figures 5(a)-5(c), respectively). According to the graphic values in Figures 5(a) and 5(b), an increase in vibration speed occurred as the speed value increased. The value of vibration was fixed and it has remained stable after reaching the speed of 3000 RPM. Even though there have been minimal fluctuations in temperature, no considerable change in the temperature value has been observed, as it can be seen in Figure 5(c). The maximum and the minimum vibration values that can be measured were between the values of $1.5 \mathrm{G}$ and $-1.5 \mathrm{G}$. The vibration values have never reached those values. The servo motor has worked with temperature under the maximum measurement of 35 degrees Celcius (Figure 5).

3.2. 3000 RPM to 0 RPM Unloaded. The servo motor was slowed down from 3000 RPM to 0 RPM with no load, and the vibration and temperature values have been recorded for 60 seconds. The vibration data taken from the vertical axis are seen in Figure 6(a), the vibration data taken from the horizontal axis are seen on Figure 6(b), and the temperature data obtained through the body are seen in the chart from Figure 6(c). According to the graphical data of Figures 6(a) and 6(b), it has been served that as the vibration value decreases, the speed value also decreases. The vibration value has remained constant after the speed value has dropped to 0 RPM and then it has been fixed. Even though there have been minimal value changes, the temperature value has decreased before the change in the value. After that, it has increased, as the motor speed has decreased (see Figure 6(c)). In this case, it is acknowledged that the generally known servo motors get warm when they operate at lower speeds. In the measurements made, depending on the measurement direction $(X, Y, Z)$, the operating values of the servo motor are compared with the reference values. From the value of the normal operation of the corresponding servo motor, depending on the measurement direction according to the references, it is observed that it works between the maximum vibration value of $1.5 \mathrm{G}$ and minimum vibration value of $-1.5 \mathrm{G}$. It has been observed that the maximum temperature value of 45 servo motor working referenced to the standard operating value of the respective servo motor works under the maximum value.

3.3. 0 RPM to 3000 RPM Loaded. The servo motor, with the label information seen, is increased from 0 RPM to 3000 RPM with on-load, and the vibration and temperature values have been recorded for 60 seconds. The vibration data have been taken on the body in the vertical axis, as seen in Figure $7(a)$, data taken on the body in the horizontal axis are illustrated in Figure 7(b), and the temperature data taken on the body in Figure 7 are shown on the graph. According to the graphical data shown in Figures 7(a) and 7(b), when the speed value reaches $500 \mathrm{RPM}$, it is observed that the vibration values exceed over the limit that can be measured. A decrease in the vibration value has been observed when the speed value of 500 RPM increases to 1500 RPM. While the 


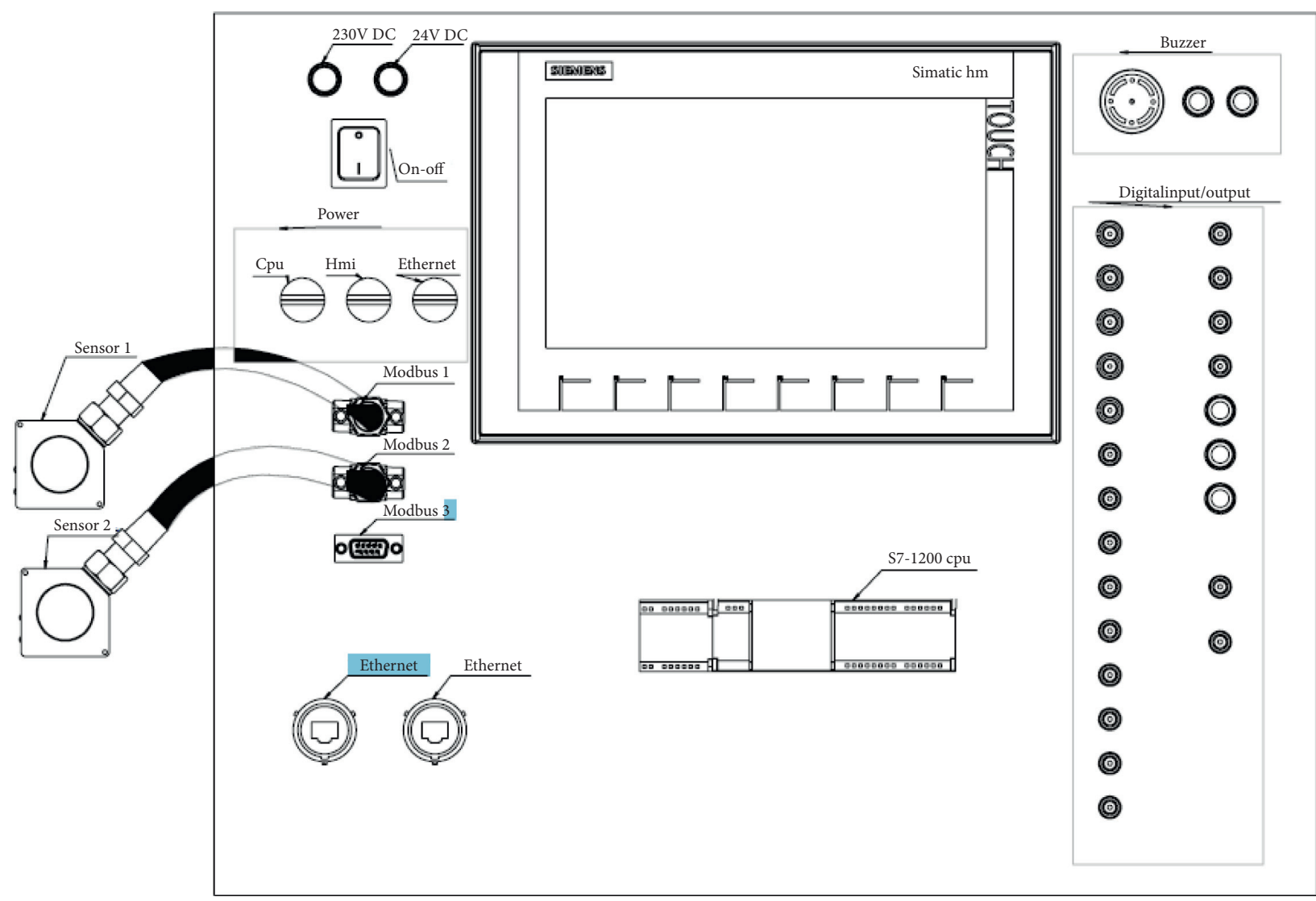

(a)

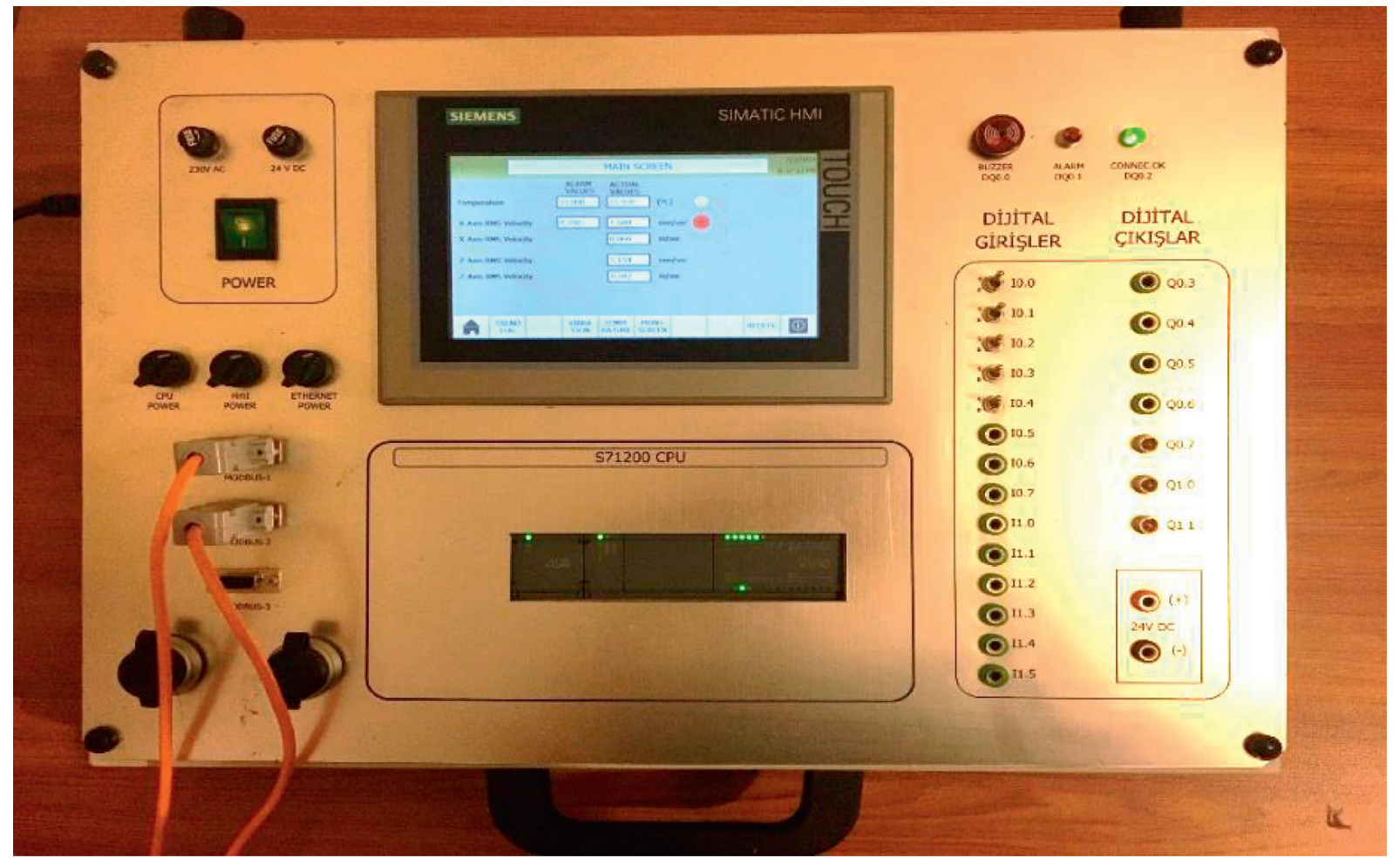

(b)

FIGURE 4: (a) Design of the vibration and temperature measurement set and its appearance at the moment of energization ((b) alternative). 


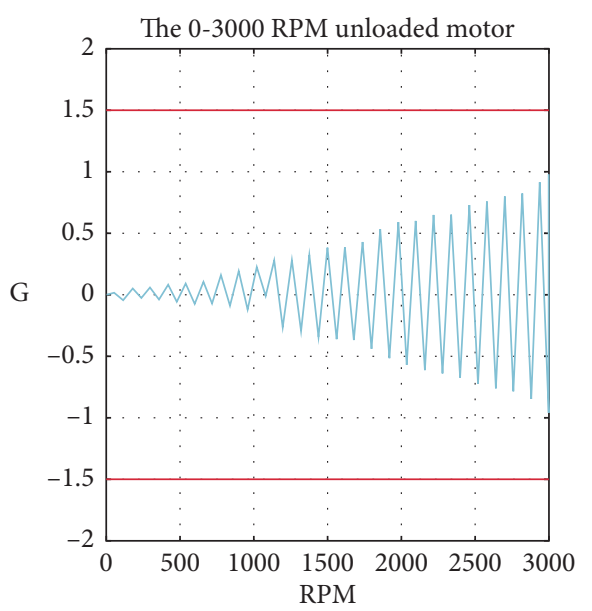

(a)

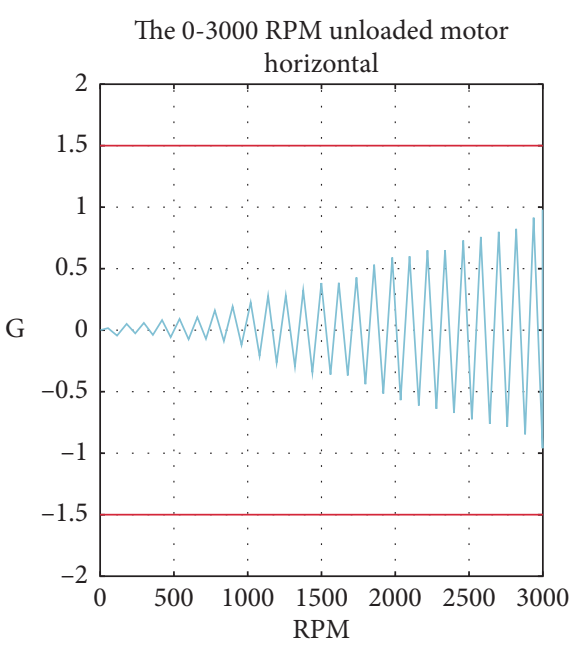

(b)

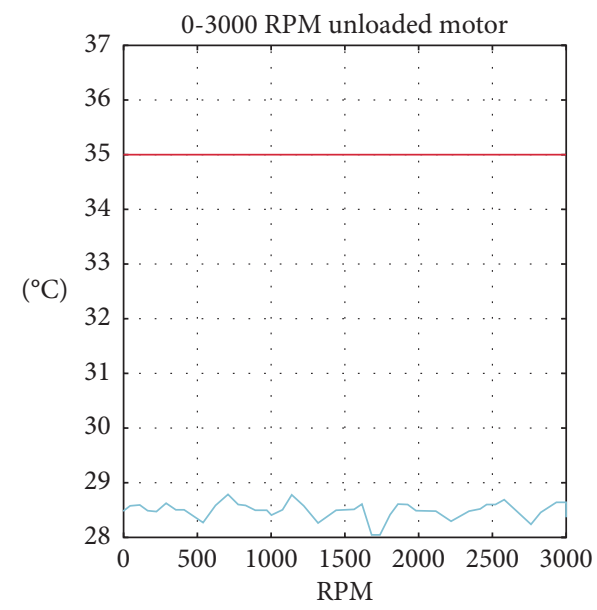

(c)

FiguRE 5: The 0-3000 RPM measurement-speed graph of the unloaded motor: (a) vertical axis, (b) horizontal axis, and (c) temperaturespeed graph of the unloaded motor.

speed value has increased from 1500 RPM to 3000 RPM, the vibration value also has increased and exceeded the limit values. As a result of the vibration values measured loaded, it can be concluded that the servo motor does not work properly loaded and that maintenance should be applied. Even though there have been minimal fluctuations in the temperature of the motor, it can be seen that it has been working in an acceptable manner, in accordance with the temperature value, as illustrated in Figure $7(\mathrm{c})$.

3.4. 3000 RPM to 0 RPM Loaded. The servo motor, with the label information seen, is increased from 0 RPM to 3000 RPM with on-load, and the vibration and temperature values have been recorded for 60 seconds. The vibration data have been taken on the body in the vertical axis, as seen Figure 8(a), data taken on the body in the horizontal axis are illustrated in Figure $8(\mathrm{~b})$, and the temperature data taken from the body are graphically shown in Figure 8(c). A decrease in the value of vibration has been observed in the graphic values in Figures 8(a) and 8(b) until the speed value has dropped from 3000 RPM to 1200 RPM. While the speed value has decreased from 1200 RPM to 700 RPM, an increase in the vibration value could have been observed, and when the speed value has reached 700 RPM, it has exceeded the limit values. While the speed value dropped from 700 RPM to $0 \mathrm{RPM}$, the vibration value also decreased and became $0 \mathrm{G}$. When the servo motor vibration values have exceeded the limit values at 700 RPM, it could have been observed that the servo motor needed maintenance. When observing the temperature graph from Figure 8(c), it can be seen that the servo motor works at typical temperature values.

\section{Discussion}

System performance may be dependent on the effects of vibration and temperature data such as the total operating time of the system, operating conditions of the system, and the external environment. So, the reference value can be obtained from an idle system. By applying the specified load, 


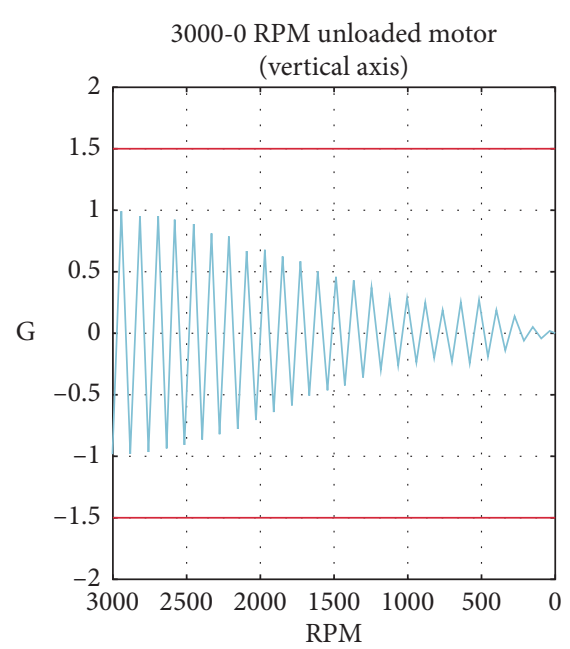

(a)

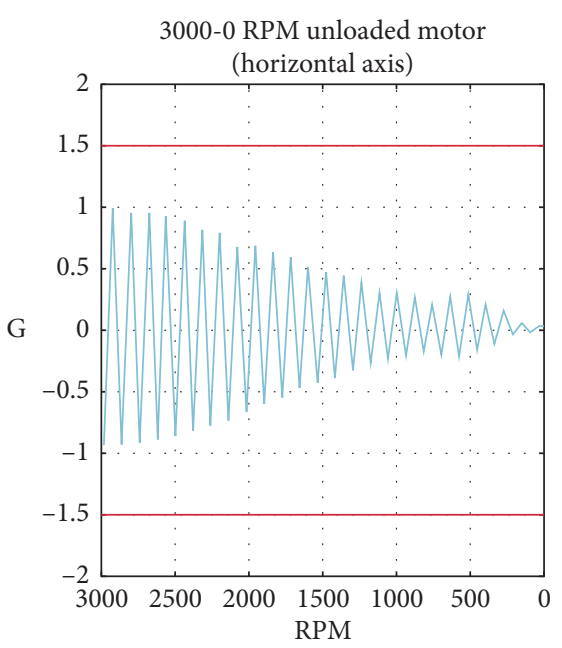

(b)

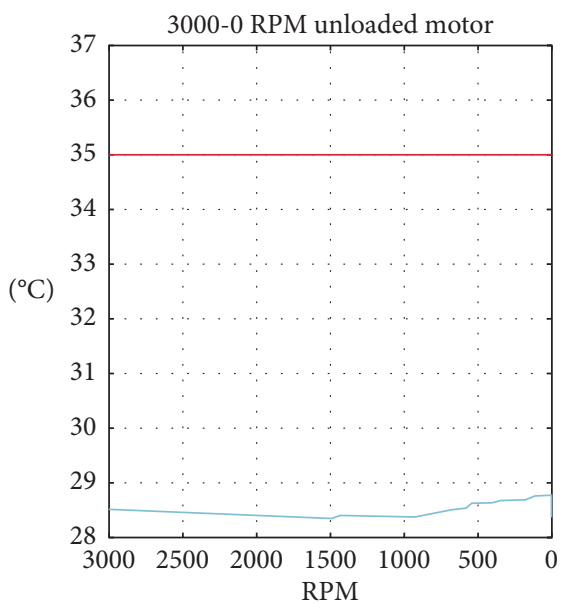

(c)

FIGURE 6: The 3000-0 RPM measurement-speed graph of the unloaded motor: (a) vertical axis, (b) horizontal axis, and (c) temperaturespeed graph of the unloaded motor.

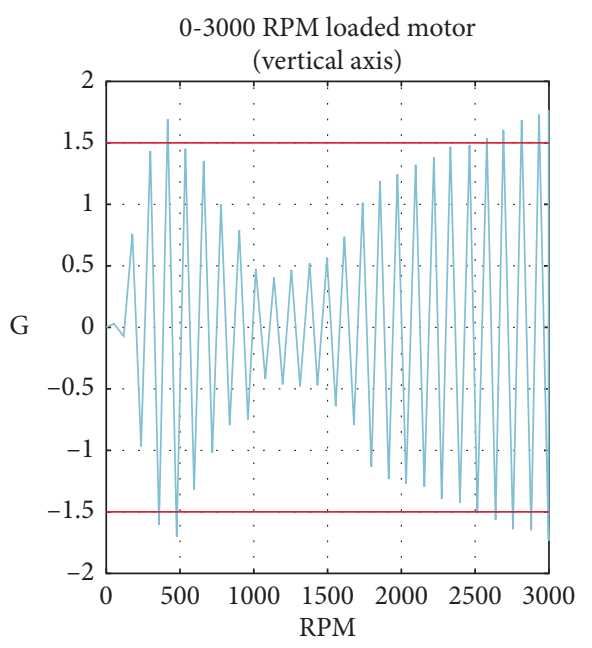

(a)

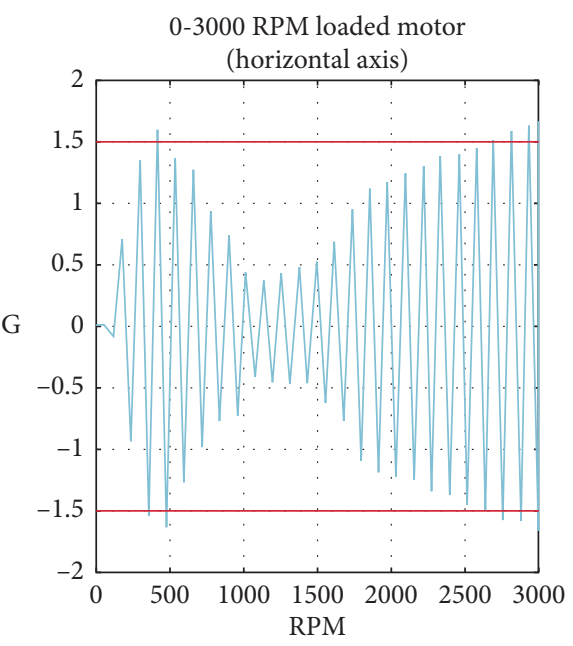

(b)

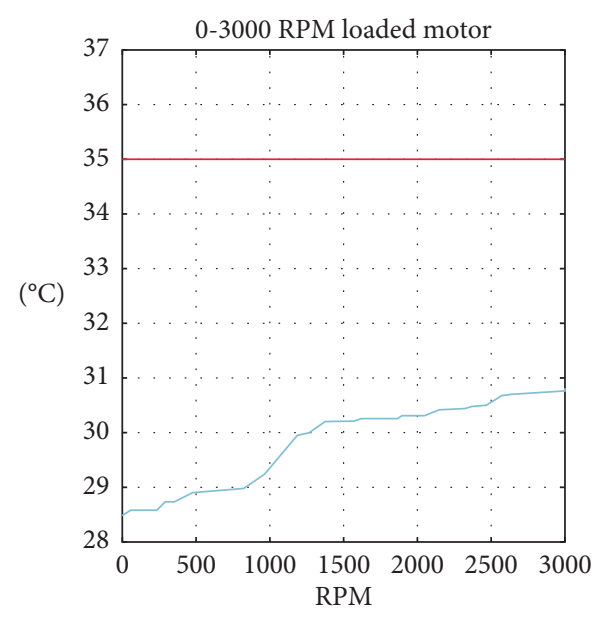

(c)

FIGURE 7: The 0-3000 RPM measurement-speed graph of the loaded motor: (a) vertical axis, (b) horizontal axis, and (c) temperature-speed graph of the loaded motor. 


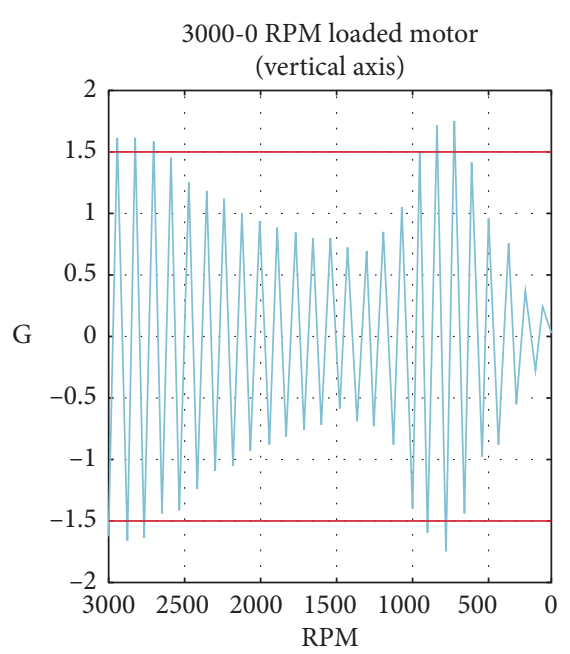

(a)

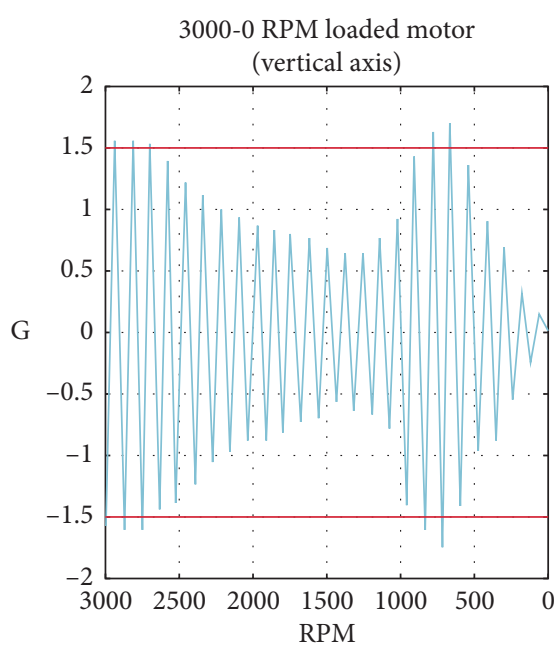

(b)

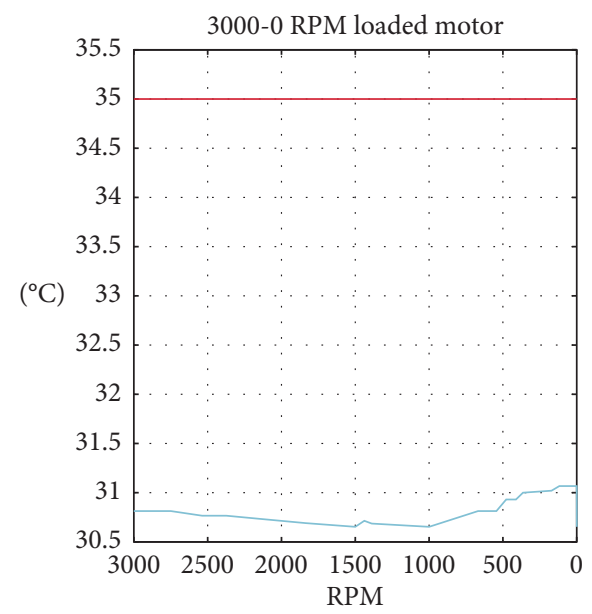

(c)

FIGURE 8: The 3000-0 RPM measurement-speed graph of the loaded motor: (a) vertical axis, (b) horizontal axis, and (c) temperature-speed graph of the loaded motor.

vibration and heat data can be monitored during operation and termination processes. As seen in the results, critical levels can be overcoming under load. This can make the framework require support before due time, and if this circumstance is not resolved in time, there may be a malfunction in the motor. Control used for detecting errors can allow it to give a warning when the limit values are exceeded and to keep values optimal.

Vibration values were measured for Siemens 1FK7063 coded servo motor at 1000, 2000, and 3000 RPM speeds, with horizontal and vertical axis displacement. The value of the temperature in degrees Celsius $\left({ }^{\circ} \mathrm{C}\right)$ has been measured for a minute and it was graphically illustrated. The working time for all the measurements has been set at $60 \mathrm{~s}$.

First, to create the sample system in terms of the reference value, data have been obtained from the system displayed in this article between 0-3000 RPM. The vibration values increase depending on the number of vibration cycles as expected. When the data has been examined, no abnormal value has been observed, and regular operations have been found according to the motor speed. However, when the heat has been measured, the reference value of $35^{\circ} \mathrm{C}$ has not been exceeded.

To test the validation of the first results of measurement made with 3000-0 RPM measurement cycles, observations were made that the vibration values have decreased according to the amount of vibration it received and it was kept within the working ranges. The temperature value at $500 \mathrm{RPM}$ is within the boundary of the first variable and the temperature value reaches the critical point in the measurement range of 3000-0 RPM. It has been identified as a normal thermal behaviour, considering the running time of this system. The values of the system were recorded while kept idle under monitoring.

A loaded system has operated between 0-3000 RPM. 0-500 RPM and 2200-3000 RPM operating ranges have exceeded the limit of the values of the vibration data. It has been identified that the vibration data have remained at the desired level from this point on. It means that the machine needs to be taken into the maintenance. Otherwise, there is a possibility of causing friction-related malfunction in the system. On the other hand, temperature values were constant at 900-1200 RPM. This variable data remains within the boundary values. The measurements in the system, based on different parameters during the study, could be done in realtime and variable areas have been identified. The data obtained from the regular study are deemed to be inside the working range. When the $0-3000$ RPM values of the motor was measured, it was observed that at 3000-2600 RPM that it exceeded over the vibration limits. The rise in these vibration values were seen at 1200-600 RPM. Between the measurements of 0-3000 RPM, the same consequences were not observed in said frequencies. This spike was observed in both measurements made with 0-3000 RPM and 3000-0 RPM and different frequencies in data are observed. Even with these vibration values, the system's temperature has never exceeded over the working temperature of 35 degrees Celsius. In conclusion, better data for predicting malfunction with 3000-0 RPM measurement method have been gained.

\section{Conclusions}

Disruptions during the mass production processes can cause a competitive disadvantage to companies in the industrial field.

Furthermore, malfunctions can cause major setbacks during the manufacturing process since continuous production depends on how well the machines can perform. Particularly in remotely controlled processes, achieving both timely and accurate monitoring might prove difficult. The system designed and developed in this study provides a way to measure the heat and the vibration in real-time. This innovative system can be configured in accordance with future research regarding industry 4.0. The monitoring system can be used on demand to show measurements for 
heat and vibrations, remotely and on demand in real-time. The control systems are integrated to provide a safe and nonhazardous production. The system can display real-time data. With the data obtained, a suitable dataset is created for use in artificial intelligence applications. Cost advantages can be provided and the system has a structure that can be improved in all aspects. It can be easily moved anywhere and has a multifunctional structure that can be used for every device and machine of similar structure. The information requested in reporting can be selected by the user. Also, by adopting the understanding of intervention before failure occurs, the highest efficiency can be obtained and the production resulting from a halt or unplanned maintenance caused by the breakdown can be contributed to production by minimizing the cost losses. This system vibration and temperature values can be examined during the operation of the machines without damaging the systems and the data that will cause problems can be monitored. In addition, it can be examined in other motors without damaging machinery and equipment, and how real errors are reflected on the graphics as a result of vibration measurements can be examined.

\section{Data Availability}

The data are available on request through a data access committee or institutional review board or from the authors by sending e-mail.

\section{Conflicts of Interest}

The authors declare that there are no conflicts of interest regarding the publication of this paper.

\section{References}

[1] N. Arthur and J. Penman, "Induction machine condition monitoring with higher order spectra," IEEE Transaction on Industrial Electronics, vol. 47, no. 5, pp. 1051-1105, 2000.

[2] H. Arslan, M. Ranjbar, E. Seçgin, and V. Çelik, "Theoretical and experimental investigation of acoustic performance of multi-chamber reactive silencers," Applied Acoustic, vol. 157, pp. 543-552, 2020.

[3] N. Johansson, E. Roth, W. Reim, and A. Baraçlı, "Smart and sustainable maintenance: capabilities for digitalization of maintenance," Sustainability, vol. 11, no. 13, p. 3353, 2019.

[4] N. Saravanan, V. N. S. K. Siddabattuni, and K. I. Ramachandran, "Fault diagnosis of spur bevel gear box using artificial neural network (ANN), and proximal support vector machine (PSVM)," Applied Soft Computing, vol. 10, no. 1, pp. 344-360, 2010.

[5] M. Pophaley and R. K. Vyas, "Plant maintenance management practices in automobile industries: a retrospective and literature review," Journal of Industrial Engineering and Management, vol. 3, no. 3, pp. 512-541, 2010.

[6] C. Chengbin, J. M. Proth, and P. Wolff, "Predictive maintenance: the one-unit replacement model," International Journal of Production Economics, vol. 54, no. 3, pp. 285-295, 1998.

[7] U. Suadiye, M. Sönmez, and Öztürk, Control of Three Dimensional Moving System Designed for Wide Field by PLC and
Observing by SCADA, AutomotionSempozium, Denizli, Turkey, 2005.

[8] R. K. Mobley, "An introduction to predictive maintenance," Plant Engineering, vol. 54, 2002.

[9] O. Sadettin and N. Aktürk, "Determination of physical faults in gearbox through vibration analysis," Journal of the Faculty of Engineering and Architecture of Gazi University, vol. 18, no. 3, 2003.

[10] H. C. Roy, Principles of Planned Maintenance, Edward Arnold, London, UK, 1974.

[11] B. P. Lientz and B. Swanson, Software Maintenance Management, Addison-Wesley Longman Publishing, Boston, MA, USA, 1980.

[12] A. S. Corder, Maintenance Management Techniques, McGraw-Hill, London, UK, 1976.

[13] H. Sun, Y. Zhang, D. Baleanu, W. Chen, and Y. Chen, "A new collection of real world applications of fractional calculus in science and engineering," Communications in Nonlinear Science and Numerical Simulation, vol. 64, pp. 213-321, 2018.

[14] A. Duyar and W. Merrill, "Fault diagnosis for the Space Shuttle main engine," Journal of Guidance, Control, and Dynamics, vol. 15, no. 2, pp. 384-389, 1992.

[15] J. Litt, M. Kurtkaya, and A. Duyar, "Sensor fault detection and diagnosis for a T700 turboshaft engine," Journal of Guidance, Control, and Dynamics, vol. 18, no. 3, pp. 640-642, 1995.

[16] A. Stetco, F. Dinmohammadi, X. Zhao et al., "Machine learning methods for wind turbine condition monitoring: a review," Renewable Energy, vol. 133, pp. 620-635, 2019.

[17] C. Li, R.-V. Sánchez, G. Zurita, M. Cerrada, and D. Cabrera, "Fault diagnosis for rotating machinery using vibration measurement deep statistical feature learning," Sensors, vol. 16, no. 6, p. 895, 2016.

[18] H. Zhengjia, S. Yudi, and Q. Liangsheng, "Rub failure signature analysis for large rotating machinery," Mechanical Systems and Signal Processing, vol. 4, no. 5, pp. 417-424, 1990.

[19] M. Behzad and M. Alvandi, "Unbalance-induced rub between rotor and compliant-segmented stator," Journal of Sound and Vibration, vol. 429, pp. 96-129, 2018.

[20] D. W. Childs and L. T. Jordan, Clearance Effects on Spiral Vibrations Due to Rubbing, ASME, New York, NY, USA, 1997.

[21] j. Yan and Y. Meng, "Industrial big data in an industry 4.0 environment: challenges, schmes, and application for predictive maintenance," IEEE Access, vol. 5, pp. 23484-23491, 2018.

[22] T. Govardhan, C. Achintya, and P. Deepak, "Numerical simulation and vibration analysis of dynamically loaded bearing with defect on rolling element," International Journal of Acoustic and Vibration, vol. 23, no. 3, pp. 332-342, 2018.

[23] S. Farhadi, "Acoustic radiation of rotating and non-rotating finite length cylinders," Journal of Sound and Vibration, vol. 428, pp. 59-71, 2018.

[24] I. Senjanovic and I. Ancic, "Validation of analytical methods for the estimation of the torsional vibration of ship power transmission system," Ocean Engineering, vol. 184, pp. 107120, 2019.

[25] E. Berber, Industrial Automatic Temperature Measurement and Control System with Microcontroller, Yildiz Technical University, Istanbul, Turkey, 2008.

[26] Q. Huang, X. Yan, C. Zhang, and H. Zhu, "Coupled transverse and torsional vibrations of the marine propeller shaft with multiple impact factors," Ocean Engineering, vol. 178, pp. $48-58,2019$.

[27] H. Jona, Implementation of a Real-Time Fast Fourier Transform on a Graphics Processing Unit with Data Streamed from a 
High-Performance Digitizer, Linköping University, Linköping, Sweden, 2015.

[28] M. Mutmaz and A. Revenga, Design Aspects of Winterized and Arctic LNG Carriers: A Classification Perspective, American Society of Mechanical Engineers (ASME), London, UK, 2006.

[29] J. I. Taylar, "Back to the basics of the rotating machinery vibration analysis," Sound and Vibration, vol. 29, no. 2, pp. 12-16, 1995.

[30] S. W. Winthrop and J. M. Smith, "Handbook of Real-Time Fast Fourier Transforms," IEEE Press, New York, NY, USA, 1995. 